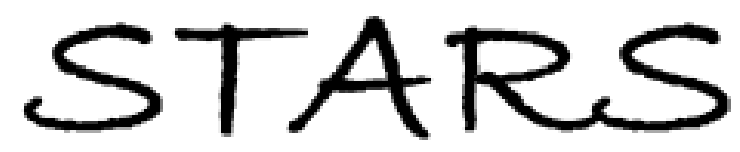

University of Central Florida

STARS

$1-1-2010$

\title{
Out-of-equilibrium characteristics of a forced translocating chain through a nanopore
}

Aniket Bhattacharya

University of Central Florida

Kurt Binder

Find similar works at: https://stars.library.ucf.edu/facultybib2010 University of Central Florida Libraries http://library.ucf.edu

This Article is brought to you for free and open access by the Faculty Bibliography at STARS. It has been accepted for inclusion in Faculty Bibliography 2010 s by an authorized administrator of STARS. For more information, please contact STARS@ucf.edu.

\section{Recommended Citation}

Bhattacharya, Aniket and Binder, Kurt, "Out-of-equilibrium characteristics of a forced translocating chain through a nanopore" (2010). Faculty Bibliography 2010s. 6999.

https://stars.library.ucf.edu/facultybib2010/6999

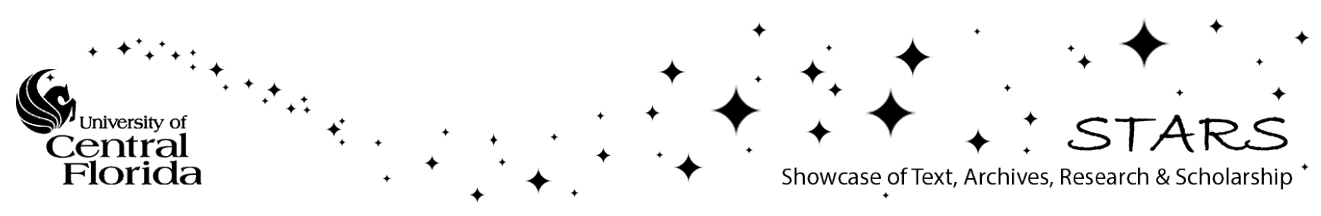




\title{
Out-of-equilibrium characteristics of a forced translocating chain through a nanopore
}

\author{
Aniket Bhattacharya $^{1, *}$ and Kurt Binder ${ }^{2}$ \\ ${ }^{1}$ Department of Physics, University of Central Florida, Orlando, Florida 32816-2385, USA \\ ${ }^{2}$ Institut für Physik, Johannes Gutenberg-Universität Mainz, Staudinger Weg 7, 55099 Mainz, Germany
}

(Received 4 December 2009; published 19 April 2010)

\begin{abstract}
Polymer translocation through a nanopore in a thin membrane is studied using a coarse-grained bead-spring model and Langevin dynamics simulation with a particular emphasis to explore out of equilibrium characteristics of the translocating chain. We analyze the out of equilibrium chain conformations both at the cis and the trans side separately either as a function of the time during the translocation process or as as function of the monomer index $m$ inside the pore. A detailed picture of translocation emerges by monitoring the center of mass of the translocating chain, longitudinal and transverse components of the gyration radii and the end to end vector. We observe that polymer configurations at the cis side are distinctly different from those at the trans side. During the translocation and immediately afterwards, the chain is clearly out of equilibrium, as different parts of the chain are characterized by a series of effective Flory exponents. We further notice that immediately after the translocation the last set of beads that have just translocated take a relatively compact structure compared to the first set of beads that translocated earlier and the chain immediately after translocation is described by an effective Flory exponent $0.45 \pm 0.01$. The analysis of these results is further strengthened by looking at the conformations of chain segments of equal length as they cross from the cis to the trans side, We discuss implications of these results to the theoretical estimates and numerical simulation studies of the translocation exponent reported by various groups.
\end{abstract}

DOI: 10.1103/PhysRevE.81.041804

PACS number(s): 36.20.-r, 87.15.H-, 87.15.A-

\section{INTRODUCTION}

Despite a large number of theoretical and numerical studies voltage driven polymer translocation through a nanopore $[1,2]$ and its prospective applications has remained an active field of research with many open questions. Initial theoretical work of Chuang Kantor and Kardar [3], Kantor and Kardar [4], Sung and Park [5], Muthukumar [6], followed by more recent theoretical studies by Dubbledam et al. [7,8], Panja et al. [9-11], Sakaue [12], and Slater et al. [13] has sparked renewed interests and numerical work [14-26]. In a recent paper [26] for the case of forced translocation we have compared predictions from theoretical studies with those obtained using Langevin dynamics simulation results [27]. We observed that the driven translocation process is dominated by out of equilibrium characteristics which may lead to misinterpretation of the time averaged data for the translocated chain. We further noticed that one needs to look at the chain segments at the cis and the trans side separately to get a true picture of the translocation process.

The purpose of our work is to collect the central facts that show the nonequilibrium character of forced translocation in its various aspects. Of course, development of a theory that puts all our observations in a coherent framework would be very desirable, but is beyond the scope of our work. However, we present some elements of a phenomenological theoretical description of nonequilibrium behavior, in terms of an effective time-dependent correlation length that describes on which scales excluded volume interactions during translocation are equilibrated. We believe that a coherent descrip-

\footnotetext{
*Author to whom the correspondence should be addressed; aniket@physics.ucf.edu
}

tion of these aspects is provided by our paper, and thus it will constitute a sound basis on which the future development of such a desirable theory can be based.

To explicitly demonstrate the out of equilibrium characteristics of relevant physical parameters are studied in detail by monitoring the trans and the cis characteristics separately as a function of the chain segments separately at the cis and trans compartments. To emphasize the role of out of equilibrium aspects we have taken a reductio ad absurdum approach. We first hypothesize that the chain undergoing forced translocation is at each instant of time in equilibrium; we then demonstrate violation of this hypothesis for the forced translocation process in several physical quantities obtained from simulation studies. Of course, we also have carried out simulation studies for the unbiased translocation so that these results can serve as reference for the specific pore-wall geometry that we choose. In the next section, we describe the model. In Sec. III, we present the results from Langevin dynamics simulation. In Sec. IV, we discuss in detail the relevance of these results

\section{MODEL}

We have used the "Kremer-Grest" bead spring model to mimic a strand of DNA [28]. Excluded volume interaction between monomers is modeled by a short range repulsive $\mathrm{LJ}$ potential

$$
U_{L J}(r)= \begin{cases}4 \epsilon\left[\left(\frac{\sigma}{r}\right)^{12}-\left(\frac{\sigma}{r}\right)^{6}\right]+\epsilon & \text { for } r \leq 2^{1 / 6} \sigma \\ 0 & \text { for } r>2^{1 / 6} \sigma .\end{cases}
$$

Here, $\sigma$ is the effective diameter of a monomer, and $\epsilon$ is the strength of the potential. The connectivity between neighbor- 
ing monomers is modeled as a finite extension nonlinear elastic (FENE) spring with

$$
U_{F E N E}(r)=-\frac{1}{2} k R_{0}^{2} \ln \left(1-r^{2} / R_{0}^{2}\right),
$$

where $r$ is the distance between consecutive monomers, $k$ is the spring constant, and $R_{0}$ is the maximum allowed separation between connected monomers. We use the Langevin dynamics with the equation of motion

$$
\ddot{\vec{r}}_{i}=-\vec{\nabla} U_{i}-\Gamma \dot{\vec{r}}_{i}+\vec{W}_{i}(t) .
$$

Here $\Gamma$ is the monomer friction coefficient and $\vec{W}_{i}(t)$, is a Gaussian white noise with zero mean at a temperature $T$, and satisfies the fluctuation-dissipation relation

$$
\left\langle\vec{W}_{i}(t) \cdot \vec{W}_{j}\left(t^{\prime}\right)\right\rangle=6 k_{B} T \Gamma \delta_{i j} \delta\left(t-t^{\prime}\right) .
$$

The purely repulsive wall consists of one monolayer of immobile LJ particles of diameter $1.5 \sigma$ on a triangular lattice at the $x y$ plane at $z=0$. The pore is created by removing the particle at the center. Inside the pore, the polymer beads experience a constant force $\mathbf{F}=F \hat{z}$ along positive $z$ direction $(F \hat{z} \neq 0$ for $|z| \leq \sigma / 2)$ and a repulsive potential from the inside wall of the pore. The reduced units of length, time and temperature are chosen to be $\sigma, \sigma \sqrt{\frac{m}{\epsilon}}$, and $\epsilon / k_{B}$, respectively. For the spring potential we have chosen $k=30$ and $R_{0}$ $=1.5 \sigma$, the friction coefficient $\Gamma=1.0$, and the temperature is kept at $1.5 / k_{B}$ throughout the simulation. Evidently with an applied bias translocation occurs along $+z$ direction while for the unbiased case the translocation can occur along $\pm z$ directions.

For the case of unbiased translocation we have chosen chain lengths $N=17,33,65,129$, and 257 and put the middle monomer symmetrically inside the pore $(z=0)$ with equal number of monomers at the cis and the trans side and let the translocation occur in either $+z$ or $-z$ directions. We have checked that for a large number of repeated trials the probability for translocation from right to left or vice versa is the same. In this case each chain is equilibrated keeping the middle monomer at the fixed location $z=0$ inside the pore and then releasing it for translocation. The results are averaged over at least for 2000 trials.

For the case of forced translocation we carried out simulations for chain lengths $N$ from 8-256 for several choices of the biasing force $F=2,4,6$, and 12 , respectively. For most cases we show the results for $F=6$. Initially the first monomer of the chain is placed at the entry of the pore. Keeping the first monomer in its original position the rest of the chain located at the cis compartment is then equilibrated for times at least an amount proportional to the $N^{1+2 \nu}$. The chain is then allowed to move through the pore along $z$ direction. Even for the forced translocation for short times back and forth motion of the chain is possible. However, a successful translocation occurs when the entire chain initially located at the cis side moves to the trans side driven by the force $\mathbf{F}$ $=F \hat{z}$ present inside the pore. When the last monomer exits the pore we stop the simulation and note the translocation time and then repeat the same for 5000 such trials. In comparison to reality, our model is drastically simplified: we deal with a neutral homopolymer without explicit solvent, there are neither hydrodynamic interactions nor long-range electrostatic forces. However, our model is of the same type as most of the currently available theoretical work. We feel that this simple limiting case needs to be understood first before more realistic cases may be tackled.

\section{RESULTS}

As mentioned in the introduction that along with the results for the forced translocation we also present results for the unbiased case, as necessary for comparison. Except for Fig. 4 where plots are shown for four different biases the remaining figures for the forced translocation refer to $F$ $=6.0$. During the translocation process we have monitored several quantities. Before we present our results here we explain the notations used. The transverse and longitudinal components of the gyration radii are denoted as $R_{g t}$ and $R_{g l}$, respectively. To study various aspects of the chain segments residing at the trans and cis sides we characterize the chain monomers with the index $m$, such that $m=1$ and $M=N$ denote the first and last beads, respectively. In the case of forced translocation initially the first bead $(m=1)$ is placed inside the pore and translocation ends when the last bead $(m=N)$ leaves the pore. In addition, we denote the number of monomers $n$ residing either at the cis or the trans side of the pore and study several quantities as a function of the number of monomers $n$. For example, $R_{g t}^{c i s}(n)$ and $R_{g l}^{c i s}(n)$ denote the transverse and longitudinal components of the gyration radii for a segment of length $n$ residing at the cis side. Likewise, $R_{g t}^{\text {trans }}(n)$ and $R_{g l}^{\text {trans }}(n)$ represents the transverse and longitudinal components of the gyration radii for a segment of length $n$ residing at the trans side, respectively. During the simulation along with these gyration radii we have monitored the mean first passage time (MFPT), the end-to-end vector $R_{N}$, location of the $z$ coordinate of the middle monomer $z(N / 2)$, and the location of the $z$ coordinate of the center of masses of the segments residing at the $\operatorname{cis}\left[Z_{C M}^{c i s}(n)\right]$ and the trans $\left[Z_{C M}^{\text {trans }}(n)\right]$ sides, respectively. In each case for a quantity $A$ the notation $\langle A\rangle$ refers to the average over 2000 5000 independent runs.

\section{A. Translocation exponent for the unbiased translocation}

First, as a reference we have checked the scaling exponents for the unbiased translocation for the choice of parameters used here. This is shown in Fig. 1 where we find $\langle\tau\rangle$ $\sim N^{2.2}$ and $\left\langle R_{g}\right\rangle \sim N^{0.61}$. Therefore, we verify that for unbiased translocation the translocation exponent $\alpha\left(\langle\tau\rangle \sim N^{\alpha}\right)$ scales as $\langle\tau\rangle \sim N^{1+2 \nu}$ as previously found by us [14] in a slightly different context using Monte Carlo (MC) and others in two dimensional $[15,18]$.

\section{B. Mean first passage time (MFPT)}

Figure 2 shows the MFPT time for a forced translocating chain as a function of the translocated segment $m$ for several chain lengths. We include this curve for future discussions as one readily gets the average translocation time $\langle\tau(m)\rangle$ of a particular monomer index $m$ or vice versa. Please note that 


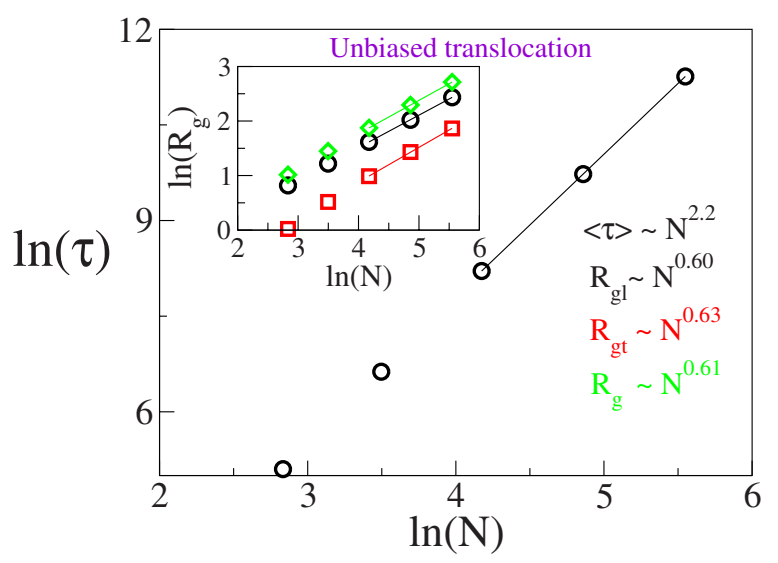

FIG. 1. (Color online) Scaling plot (log-log scale) of the mean translocation time $\langle\tau\rangle$ for the case where no bias force $F$ is applied, as a function of chain length $N$. The inset shows the corresponding plots for the gyration radii where the diamonds, circles, squares, represent the gyration radii, and the longitudinal and the transverse components $R_{g l}$ and $R_{g t}$, respectively.

for forced translocation at the beginning and end of the translocation the monomer index has a value $m=1$ and $N$, respectively, therefore, a large value of $m$ relates to a relatively a late stage of the translation process and vice versa. For larger chain lengths the nonlinear nature in the form of an $S$ shaped curve becomes more prominent where we notice that the curvature is negative (concave) for $m<N / 2$ and becomes positive for $m>N / 2$. The last few beads take relatively shorter time to translocate.

\section{What is the location of $Z_{C M}(N / 2)$ ?}

If the translocating chain were in local equilibrium then one would expect that when the chain is half way through the
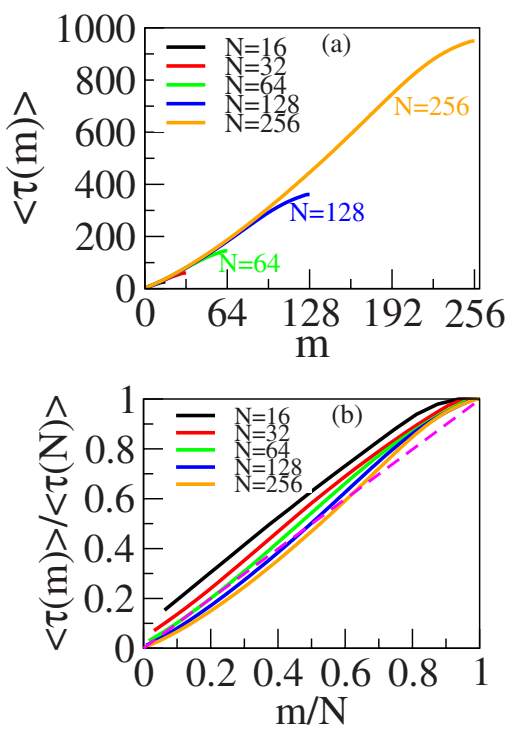

FIG. 2. (Color online) (a) MFPT for forced translocating chains. Different colors correspond to $N=16$ (black), $N=32$ (red), $N=64$ (green), $N=128$ (blue) and $N=256$ (orange). (b) MFPT normalized by the $\langle\tau(N)\rangle$ as a function of normalized chain segment $m / N$ for $N=16$ (top curve) to $N=256$ (bottom curve). The dashed straight line (magenta) corresponds to the line of unit slope for comparison

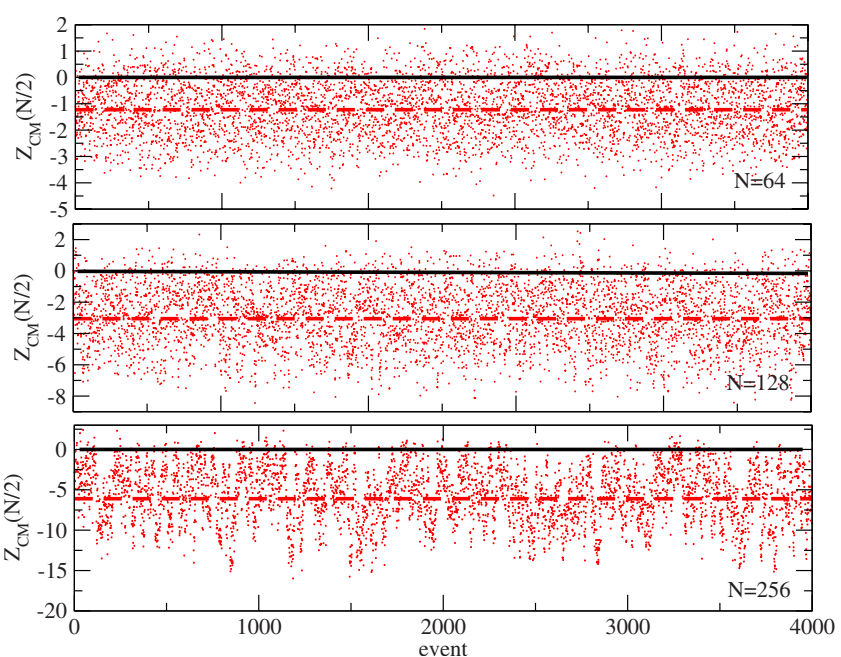

FIG. 3. (Color online) Location of the $Z_{C M}(N / 2)$ for several different chain lengths plotted vs the index labeling the individual simulation runs. The dots represent data points from each run. The dashed red line correspond to the $\left\langle Z_{C M}(N / 2)\right\rangle$ averaged over all these points. The position of $Z=0$ is denoted by the solid black line for easy comparison.

translocation process the position of the $z$ component of the center of mass $Z_{C M}(N / 2)$ should be located symmetrically with respect to the cis and the trans side. This should correspond to $z=0$ for our choice of coordinate system. Figure 3 shows the distribution of the location of $Z_{C M}(N / 2)$ and the average value $\left\langle Z_{C M}(N / 2)\right\rangle$ for three different chain lengths. Please note that while calculating $\left\langle Z_{C M}(N / 2)\right\rangle$ we have taken into consideration the back and forth motion of the chain. Therefore, here $\left\langle Z_{C M}(N / 2)\right\rangle$ implies, first, to determine the average of $Z_{C M}(N / 2)$ from a single run considering all the events when the monomer index $m=N / 2$ inside the pore and then taking average over all different runs. We note that with increasing chain length the location of $Z_{C M}(N / 2)$ goes deeper in the cis side of the chain. When we plot $\left\langle Z_{C M}(N / 2)\right\rangle$ as a function of the chain length $N$ shown in Fig. 4 it appears that after a certain crossover length the distance increases approximately linearly with the chain length for larger chains. It also appears that this crossover length increases as the bias becomes small. This implies that finite chain lengths

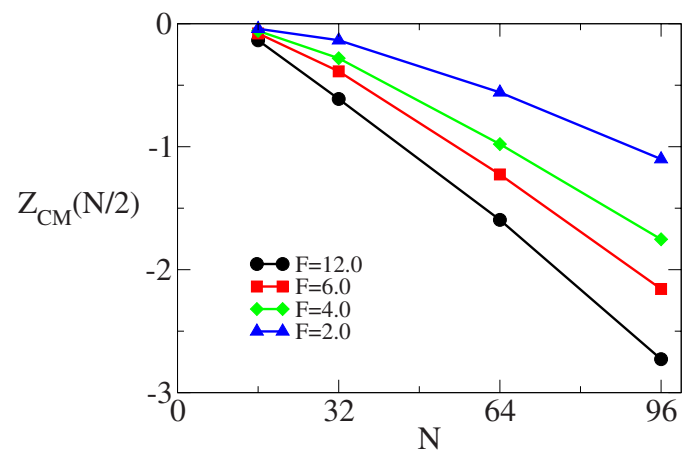

FIG. 4. (Color online) Plot of $Z_{C M}(N / 2)$ as a function of chain length for several different bias values. The circles, squares, diamonds, and triangles correspond to the bias values $F=12.0, F$ $=6.0, F=4.0$, and $F=2.0$, respectively. 

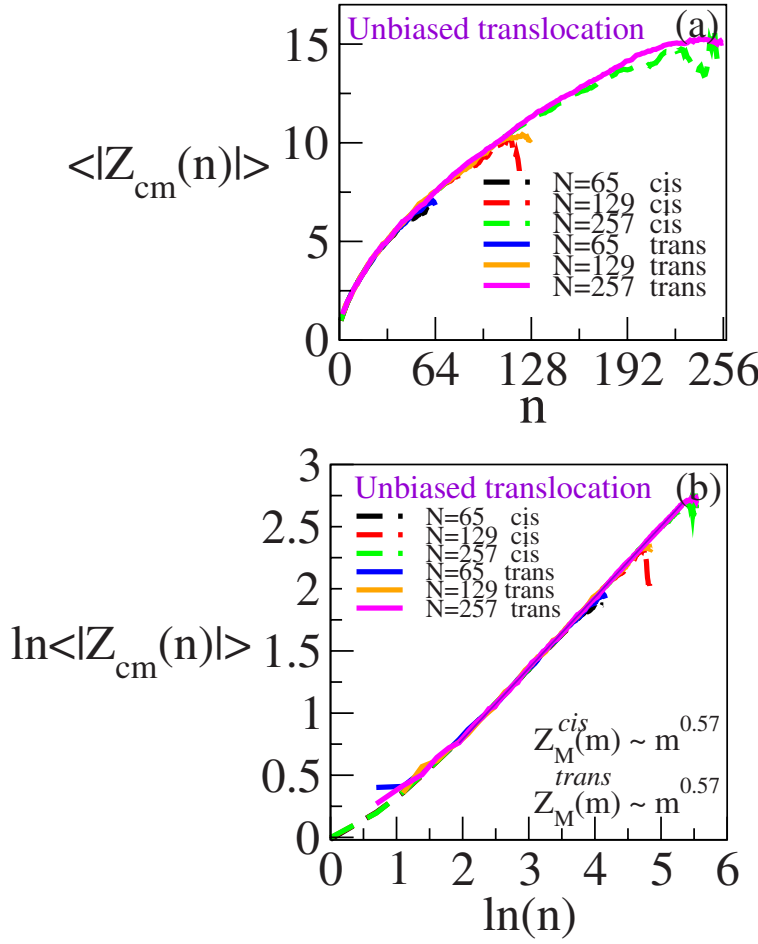

FIG. 5. (Color online) (a) Plot of $\left\langle Z_{C M}^{c i s}(n)\right\rangle$ (dashed black, red, and green) and $\left\langle Z_{C M}^{\text {trans }}(n)\right\rangle$ (solid blue, orange, and magenta) as a function of the segments on the cis and trans side for chain lengths $N=65,129$, and 257, respectively, for unbiased translocation. (b) the corresponding plot on the log-log scale where we notice that for a large fraction of the cis and trans segments $\left\langle Z_{C M}(n)\right\rangle \sim m^{0.57}$.

effects become progressively smaller as a function of the increasing bias, a result that seems counterintuitive. However, with the limited data available at this time we do not make a strong claim, but indicate to such a trend. In order to establish more accurately the exact location of a possible crossover, it will require runs with many more values of bias and longer chains making the simulations prohibitively long.

\section{Behavior of $Z_{C M}^{\text {cis }}(n)$ and $Z_{C M}^{\text {trans }}(n)$}

This asymmetry in the location of $\left\langle Z_{C M}(N / 2)\right\rangle$ becomes more obvious when we look at the absolute values of positions of the center of masses on the cis side $\left|Z_{C M}^{c i s}(n)\right|$ and the trans side $\left|Z_{C M}^{\text {trans }}(n)\right|$ separately. Again, if the local equilibrium condition should hold, one would expect that as a function of the trans and cis segments the behavior of $\left|Z_{C M}^{\text {trans }}(n)\right|$ and $\left|Z_{C M}^{i s}(n)\right|$ should be the same. For comparison, and as a reference we first show the plot for the case of unbiased translocation in Fig. 5 where we notice that the longitudinal and the transverse components of the gyration radii for both the cis and trans segments are almost indistinguishable, as anticipated. We further note that for large portion of the chain except for very small and large values of the monomer index $\left\langle\left|Z_{C M}^{\text {cis,trans }}\right|\right\rangle \sim m^{0.56}$. If the configurations of the chains in the case of unbiased translocation just realize a sequence of equilibrium configurations, we expect $\left\langle\left|Z_{C M}^{\text {cis,trans }}\right|\right\rangle \sim m^{\nu}$ with $\nu \approx 0.59$ the equilibrium Flory exponent, when $m \rightarrow \infty$. Although the effective exponent (0.56) is somewhat smaller,
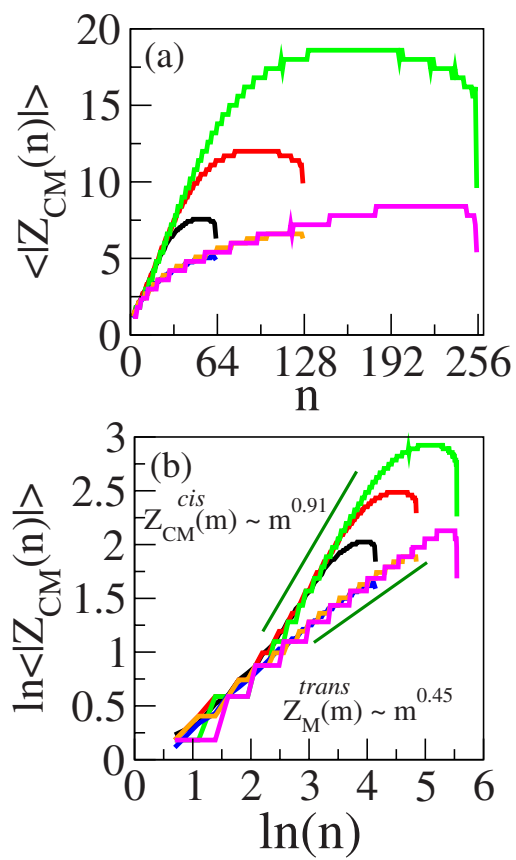

FIG. 6. (Color online) (a) Plot of $\left\langle Z_{C M}^{c i s}(n)\right\rangle$ (black, red, and green) and $\left\langle Z_{C M}^{\text {trans }}(n)\right\rangle$ (blue, orange, and magenta) as a function of the segments on the cis and trans side for chain lengths $N=64,128$, and 256, respectively, for forced translocation. Note that the data for $\left\langle Z_{c m}^{\text {trans }}(n)\right\rangle$ for different $N$ coincide almost perfectly on a common curve, so one can hardly distinguish the individual values for $m$ $<64$ on this plot; (b) the corresponding plots on log-log scale. Straight lines indicate the power laws quoted in the figure.

it is clear that in this case the translocating chains are close to equilibrium. This should be contrasted with the corresponding case for the forced translocation where we observe a very different behavior as shown in Fig. 6 for the cis and the trans segments. Since the $\left\langle Z_{C M}^{\text {cis }}(n)\right\rangle$ takes negative values at the $c i$ side we consider the absolute values $\left\langle\left|Z_{C M}^{c i s}(n)\right|\right\rangle$ and $\left\langle\left|Z_{C M}^{\text {trans }}(n)\right|\right\rangle$. Using Fig. 2 one can translate the figure from the variable $m$ to the corresponding MFPT. One notices that the cis component increases almost linearly for $0<m$ $<N / 2$ and scales as $\left\langle\left|Z_{C M}^{c i s}(n)\right|\right\rangle \sim m^{0.91}$, whereas the trans part after a linear rise for small $m$, increases at a much slower rate compared to the cis part. We further notice $\left\langle\left|Z_{C M}^{\text {trans }}(n)\right|\right\rangle$ $\sim m^{0.45}$, the value of the slope $(0.45)$ in this case exactly half of the corresponding value of the cis part. We notice similar qualitative behavior for $\left\langle R_{g}^{\text {cis }}(n)\right\rangle$ and $\left\langle R_{g}^{\text {transs }}(n)\right\rangle$ and defer the physical explanation of this behavior to Sec. III F.

\section{E. Gyration radii: time dependence}

To get a better idea of the out of equilibrium characteristics of the forced translocating chain we have compared the time dependence of gyration radii with those for the unbiased chain shown in Fig. 7(a). For unbiased translocation $\left\langle R_{g l}\right\rangle$ $>\left\langle R_{g t}\right\rangle$ is due to the presence of the wall that breaks the isotropy symmetry and gyration radius along the translocation direction becomes larger when calculated for the entire chain. This anisotropy occurs already for a polymer chain anchoring with a chain end at a flat impenetrable surface (a 

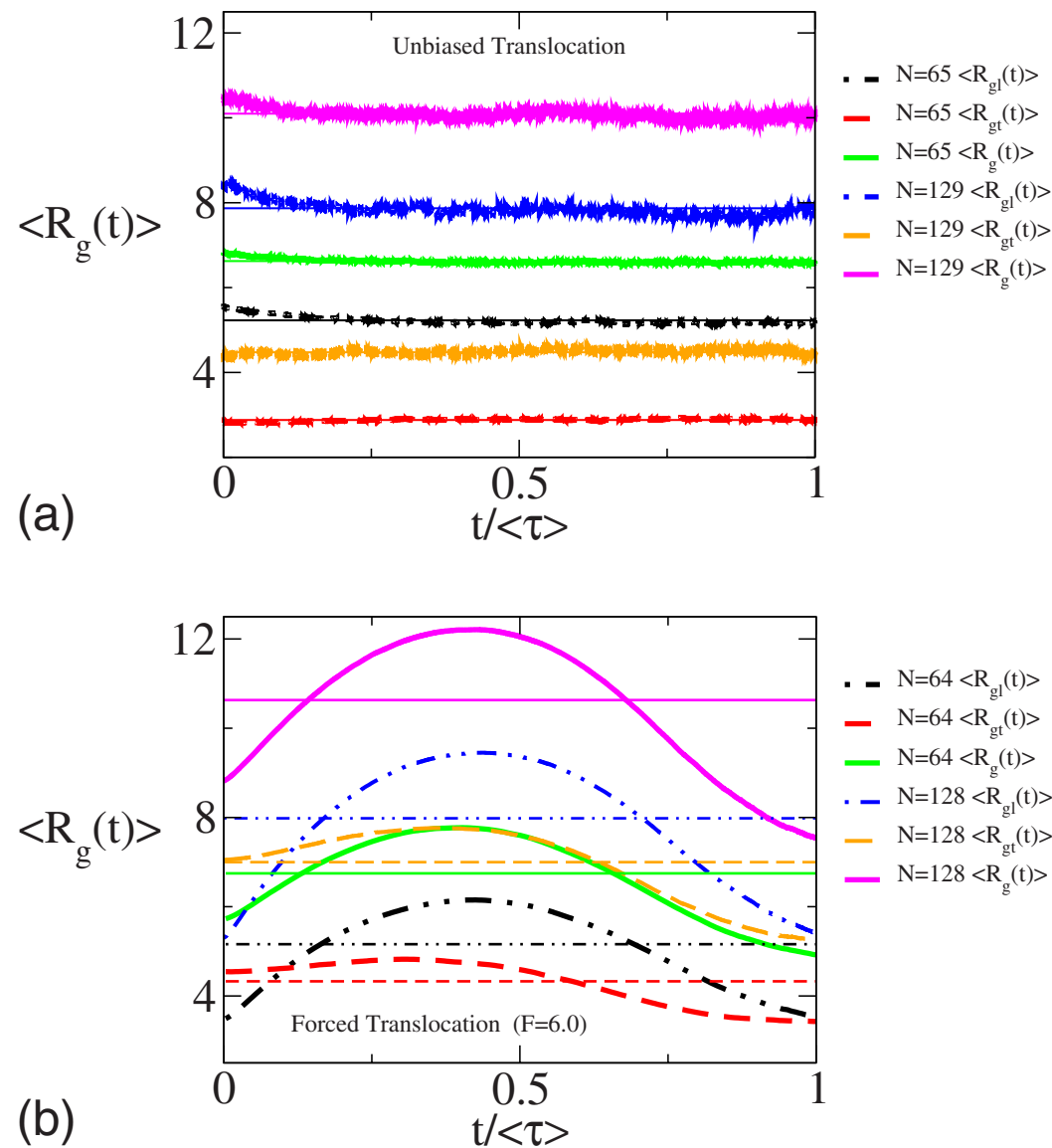

FIG. 7. (Color online) Plot of gyration radii $\left\langle R_{g}(t)\right\rangle$ as a function of time for the unbiased (a) translocation and (b) for the forced translocation where a significant variation in the size of the translocating chain is immediately noticeable. In each figure that double-dotted (black and blue) lines represent the longitudinal components, wide dashed (red and orange) lines represent the transverse components, and the solid lines (green and magenta) correspond to the gyration radii for chain lengths 65 (64) and 129 (128) for the unbiased (biased) translocation respectively. The thin straight lines in each case (with the same symbols) correspond to the corresponding time averaged values. Note that $\left.\left\langle R_{g}(t)\right\rangle\right\rangle\left\langle R_{g l}(t)\right\rangle$ $>\left\langle R_{g t}(t)\right\rangle$ for unbiased translocation. "polymer mushroom"). For unbiased translocation, the typical chain conformations are those of equilibrium mushrooms containing $m$ and $N-m$ segments, respectively. When we compare these time dependencies with those for the chain undergoing forced translocation we notice that the forced chain undergoes considerable variation in its size. However, despite this large variation in size when we calculate the average gyration radii we find $\left\langle R_{g}\right\rangle \sim N^{\nu}$ [26]. Therefore, if one does not plot and see this explicit time dependence, one would tend to make an assumption that only one bead of the chain is driven inside the pore, and therefore the chain is still described by the equilibrium Flory exponent [4]. The argument that immediately comes to ones mind that the variations are cancelled [Fig. 7(b)] which we find is wrong and it is inappropriate to describe the chain by a single exponent. In the next subsection, we will provide the correct physical reason why the chain is still described by the equilibrium Flory exponent.

\section{F. Gyration radii components at the cis and trans side}

To further demonstrate the out of equilibrium aspects of the driven translocating chain we have monitored the time dependence of the transverse and longitudinal components of the cis and trans part of the gyration radii separately and compared these characteristics with those for the unbiased translocation. Figure 8 shows the plots of the transverse and longitudinal components for the cis side $\left(\left\langle R_{g t}^{\text {cis }}(n)\right\rangle,\left\langle R_{g l}^{c i s}(n)\right\rangle\right)$ and for the trans side $\left[\left\langle R_{g t}^{\text {trans }}(n)\right\rangle,\left\langle R_{g l}^{\text {trans }}(n)\right]\right.$ separately for the unbiased translocation. As expected, except for the few first and last beads (which may depend on the local condition) the conformations for the cis and trans parts are the same [29]. We further notice that for small values of segments $m R_{g l}>R_{g t}$; however this difference might arise due to finite chain length that vanishes for large values of $m$. Within our error limits, these data are compatible with a scaling of all these radii according to a law $R \propto N^{\nu}$ with $\nu \propto 0.59$ being the expected Flory exponent.

For the case of forced translocation (Fig. 9) one immediately notices that the $\left\langle R_{g}(n)\right\rangle$ of the chain on the cis and trans sides are described by different effective Flory exponents. The effect is most pronounced for the longitudinal component. We have calculated the slopes for $N / 8<m<N / 4$ and find that $\left\langle R_{g l}^{\text {trans }}(n)\right\rangle \sim m^{0.6}$ and $\left\langle R_{g l}^{\text {cis }}(n)\right\rangle \sim m^{0.8}$. This result is consistent with the recent theory proposed by Sakaue [12] based on the propagation of tension along the chain. During the forced translocation process at early time $(m \ll N)$ the translocated monomers relax faster and are described roughly by the equilibrium Flory exponent. However, during the short time needed that a small number of $n$ monomers are pulled through the pore by the force, the remaining $N-n$ segments do not have enough time to equilibrate their configuration. One can think of this effect as the size of $R_{g}^{\text {cis }}(N$ $-n)$ remaining the same as $R_{g}^{c i s}(N)$ but with a few less number of monomers because of those that have translocated. This leads to a very weak dependence of $R_{g}^{\text {cis }}(n)$ for $n \sim N$ (a saturation observed in Fig. 9) and makes the effective Flory exponent higher on the cis side at the early stage of the translocation process. As time proceeds the number of mono- 


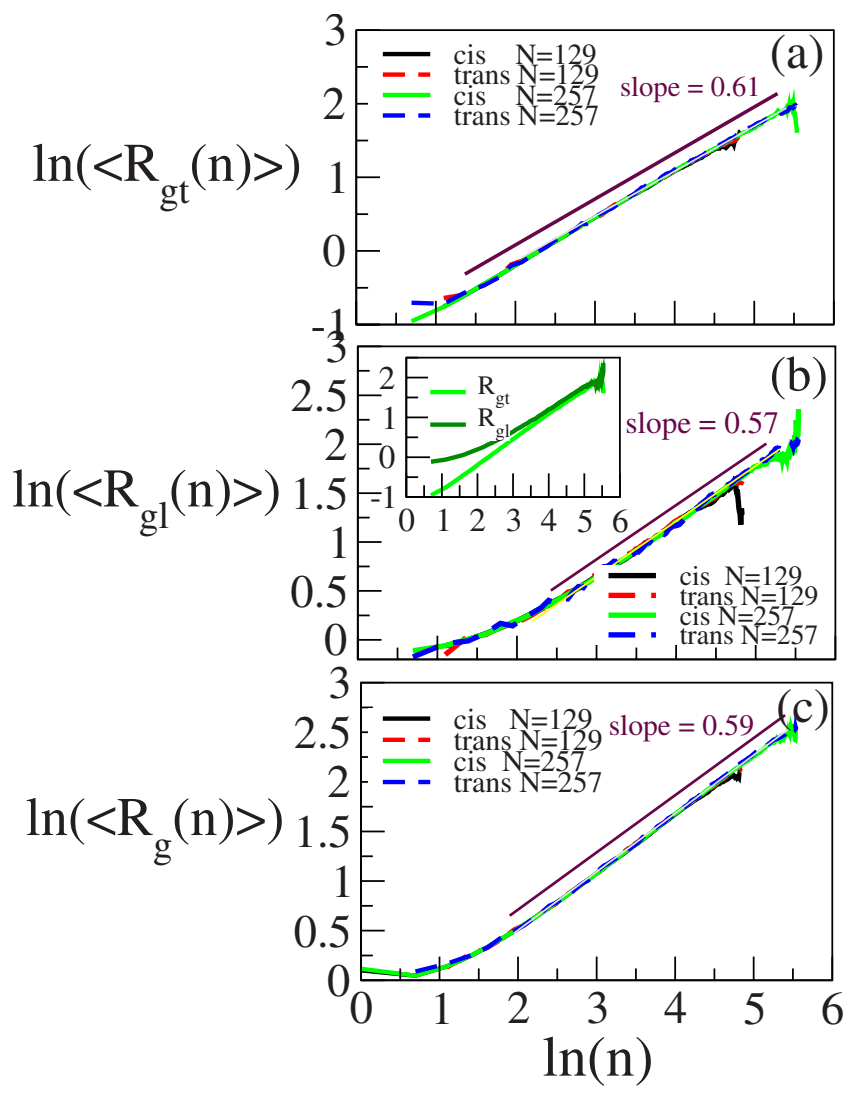

FIG. 8. (Color online) Plot of (log scale) (a) $\left\langle R_{g t}^{\text {cis }}(n)\right\rangle$, $\left\langle R_{g t}^{\text {trans }}(n)\right\rangle,(\mathrm{b})\left\langle R_{g l}^{\text {cis }}(n), R_{g l}^{\text {trans }}(n)\right\rangle$, and (c) $\left\langle R_{g}^{\text {cis }}(n)\right\rangle,\left\langle R_{g}^{\text {trans }}(n)\right\rangle$ as a function of the segments $m$ on the cis and trans sides, respectively, for the case of unbiased translocation. The straight line (purple) in each figure refers to the slope. The inset of (b) compares the longitudinal component (upper curve) $\left\langle R_{g l}(n)\right\rangle$ and the transverse component (lower curve) $\left\langle R_{g t}(n)\right\rangle$ for chain length $N=129$ for comparison.

mers on the cis side also decreases causing the slope (Fig. 9) also to decrease. This difference in behavior of $R_{g}^{\text {trans }}(n)$ and $R_{g}^{c i s}(n)$ as manifested in our simulation studies should serve as useful information for the development of detailed analytical theories of forced polymer translocation through a nanopore. Since the translocation time in the case of forced translocation for large $N$ is much smaller than the Rouse time needed to equilibrate a chain, the conformations of the chains that just have translocated are much too compact (Fig.

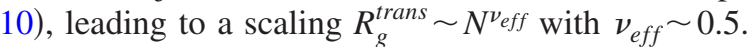

\section{G. Analysis of the subchain relaxation on cis and trans sides}

In order to get a better picture of the chain relaxation at the trans and cis side we have further investigated the relaxation of subchain conformations during the translocation process. Since this analysis requires a relatively longer chains we will restrict our discussion for chain of lengths $N=128$ and 256 consisting of eight subchains each of length 16 (for $N=128$ ) or 32 (for $N=256$ ), respectively. For the rest of the discussion in this section let's consider the chain length $N$ $=256$ as the qualitative features for the $N=128$ chain with eight subchains each consisting of 16 monomers are very

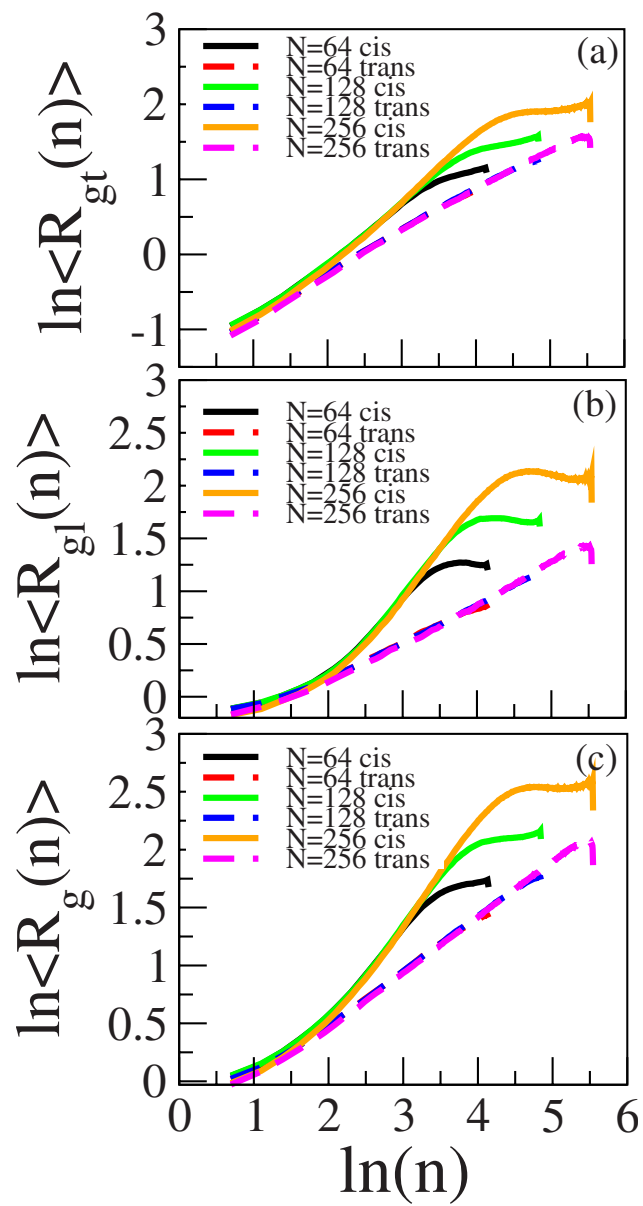

FIG. 9. (Color online) Plot of (log scale) (a) $\left\langle R_{g t}^{\text {cis }}(n)\right\rangle$, $\left\langle R_{g t}^{\text {trans }}(n)\right\rangle$, (b) $\left\langle R_{g l}^{\text {cis }}(n), R_{g l}^{\text {trans }}(n)\right\rangle$, and (c) $\left\langle R_{g}^{\text {cis }}(n)\right\rangle,\left\langle R_{g}^{\text {trans }}(n)\right\rangle$ as a function of the segments $m$ on the cis and trans sides, respectively. In each figure the solid and dotted lines correspond to the cis and trans components, respectively. Note that the curves for the trans part (lower set of dashed curves in each part of the figure) superimpose to such a large extent that they are hardly distinguishable from the data for $N=256$ in these figures.

similar. Let us denote these subchains of length 32 as $n_{i}$ $(i=1,2, \cdots 8)$. Therefore the $i$-th chain segment $n_{i}$ consists of monomers having indices from $(i-1) * 32+1$ to $i * 32$. At the start of the translocation process initially, all subchains are in equilibrium and have their values for their end-to-end distance and gyration radius components as appropriate for a calculation done for the mushroom in equilibrium, which is not yet translocating and the entire chain being located at the cis side. Once the translocation starts, we study all these subchains at times when the last monomer of each segment $n_{i}$ has just translocated from the cis to the trans side. Therefore, for the chain length $N=256$ consisting of eight subchains, at the end of a successful translocation process we will have eight different subchain conformations starting with $n_{\text {trans }}=1$ and $n_{\text {cis }}=7$ until all the segments are translocated when $n_{\text {trans }}=8$ and $n_{\text {cis }}=0$. We then analyze these subchain conformations averaged over 2000 repeated trials.

It is expected that the subchain $n_{i}$ which has just translocated and its two neighboring subchains $n_{i-1}$ and $n_{i+1}$ will be affected by the driving force and out of equilibrium charac- 


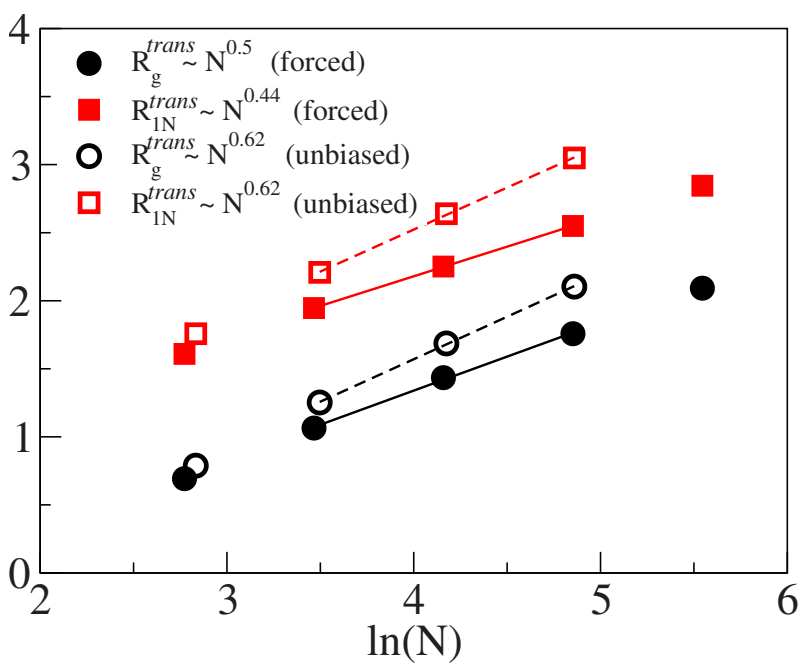

FIG. 10. (Color online) Plot of the radius of gyration $\left\langle R_{g}\right\rangle$ and end to end distance $\left\langle R_{N}\right\rangle$ (logarithmic scale) of the chain which has just translocated as a function of the chain length $N$ (logarithmic scale). The solid (open) circles (black) and squares (red) refer to the forced (unbiased) translocation.

teristics will dominate most. But the chain segments which are further away will progressively be closer to equilibrium. In other words, by the time the subchain $n_{i}$ has just translocated, the translocated chain segments $n_{1}, n_{2}, n_{3} \cdots n_{i-2}$ will have more time to relax (as a function of the decreasing segment index $i$ ) compared to the segment $n_{i-1}$, while the segments $n_{8}, n_{7} \ldots$ will remain mostly in equilibrium and hardly feel that the subchain $n_{i}$ has just translocated. Thus if we look at the conformations for all the segments at these times we expect to see that the cis subchain closest to the pore is mostly stretched with a higher value of the Flory exponent, while the trans subchain which just finished translocation is compressed most with a lower value of the Flory exponent. These higher (lower) value at the cis (trans) side will progressively taper toward the equilibrium value on either side of the pore. The increased value of the Flory exponent will appear like a propagating defect.

In the accompanying Fig. 11 (subchain length 32) we have plotted the gyration radii and the corresponding transverse and the longitudinal components for the chain segments at times immediately after subchains $1,2, \cdots 8$ have translocated. We have carried out the same analysis (not shown here) for a chain length $N=128$ for subchain lengths 16. This enables us to extract the effective Flory exponents $\nu_{\text {eff }}$ for each point in Fig. 11 calculated from the gyration radii for subchain lengths 32 (for $N=256$ ) and 16 (for $N$ $=128$ ), respectively. This is shown in Fig. 12. In Figs. 11 and 12 we kept the vertical scale the same for all the graphs for ready comparison of the gyration radii and the $\nu_{\text {eff }}$ at different stage of the translocation process. First, one notices that after the translocation process, the chain is still compressed. Second, at the trans side the slope of the line connecting the points increases monotonically (not shown) from the left to the right, the gyration radii of the trans segment closest to the pore being the least [such as in Figs. 11(f) and 11(g)]. This is due the fact, as stated above, that the farthest trans

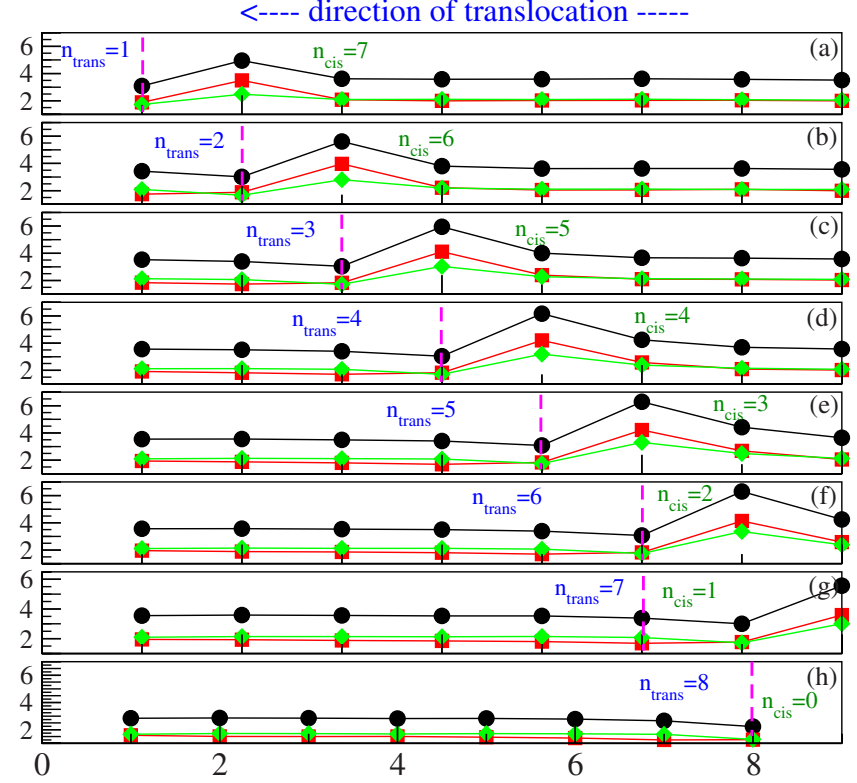

FIG. 11. (Color online) Plot of gyration radii for the chain segments of length $n=32$ for chain length $N=256$ at different stages of the translocation process when integral number of chain segments $n_{i}$ have translocated. The vertical red lines differentiate the trans and the cis segments. The filled squares and diamonds represent the longitudinal and the transverse components of the gyration radii, respectively, and the filled circles represent the gyration radii.

segment have more time to approach equilibrium configuration from its initial compressed state at the entry of trans side. Likewise, on the cis side the slope of the curve joining the cis segments decreased monotonically for the same reason that the farthest segment is still in equilibrium and the cis

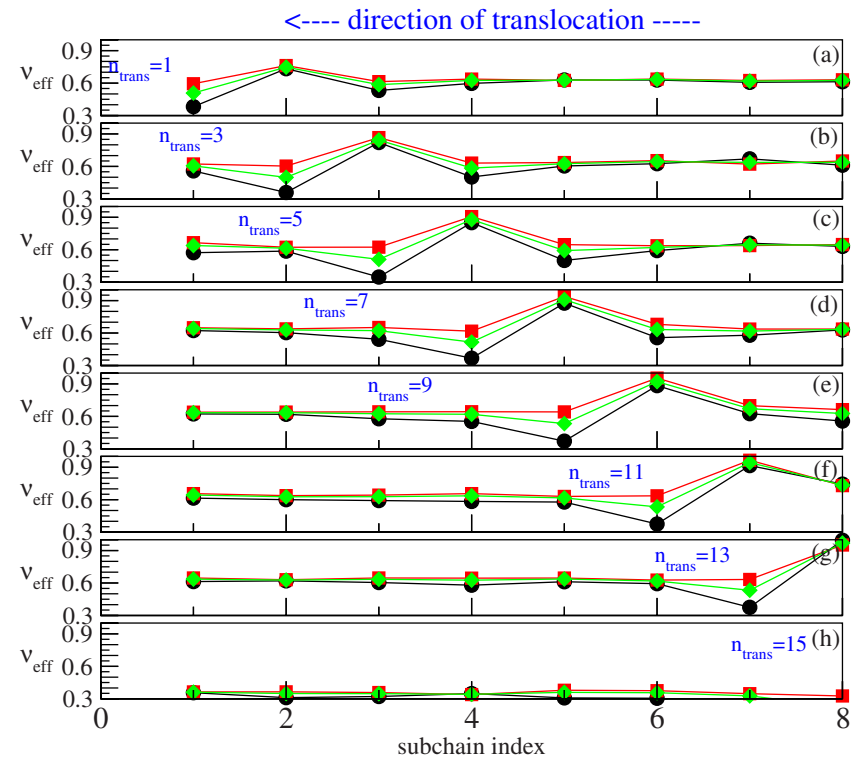

FIG. 12. (Color online) Plot of effective Flory exponents as a function of the subchain index at different stage of the translocation process. The filled squares and diamonds represent the effective exponents for the longitudinal and the transverse components of the gyration radii respectively and the filled circles represent the effective exponent for the gyration radii. 

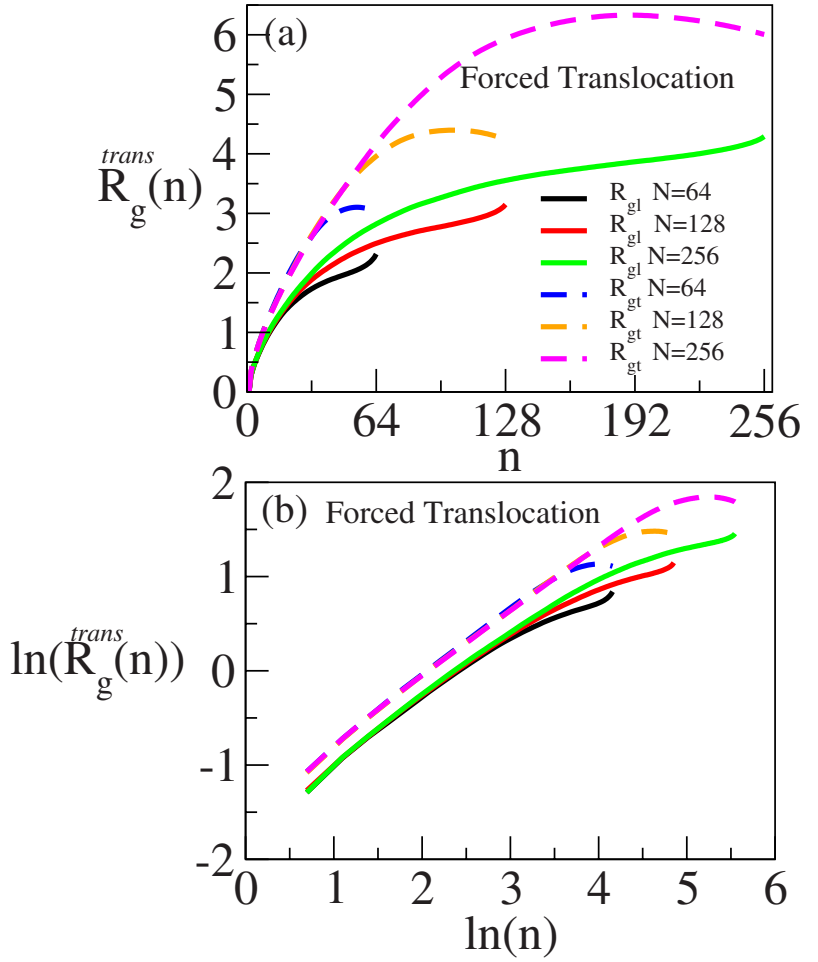

FIG. 13. (Color online) (a) Plot of longitudinal (solid lines) and transverse (dotted lines) gyration radii for the polymer which has just translocated as a function of the monomer index $m$. The solid black, red, and green lines and dotted blue, orange, and magenta lines correspond to the chain lengths $N=64,128$, and 256 for the longitudinal and transverse components, respectively. (b) The same plot on a log-log scale.

segment closest to the pore is mostly stretched. Finally, from Fig. 12 we indeed notice that the effective Flory exponent is large near the pore and the nonmonotonic behavior looks like a propagating defect. It is clear from the data that subchain relaxation proceeds exactly the way as contemplated in above.

\section{H. Properties of a translocated chain}

In order to interpret the properties of a chain which has just translocated, we introduce the concept of a timedependent correlation length $\xi(t)$ describing the range over which (on the trans side of the membrane) excluded volume effects are established (at a time $t$ after the translocation process has begun). We start from the fact that for the Rouse model with excluded volume interactions the length (chain radius $R$ ) and relaxation time $\tau$ are related as $\tau \propto R^{z} \propto N^{\nu z}$ $=N^{2 \nu+1}$ where the dynamic exponent $z=2+1 / \nu$. Consider now the "Gedankenexperiment" that for a Gaussian chain at time $t=0$ the excluded volume interactions are suddenly switched on. Then the chain starts to swell, its radius will grow from the Gaussian size $\left(R \propto N^{1 / 2}\right)$ to the swollen size $\left(R \propto N^{\nu}\right.$ with $\left.\nu \approx 0.59\right)$ during the time $\tau$. At time $t$, one can envisage this gradual swelling that on a length scale $\xi(t)$ excluded volume has already been established, but not on scales much larger than $\xi(t)$; so the chain is a Gaussian string
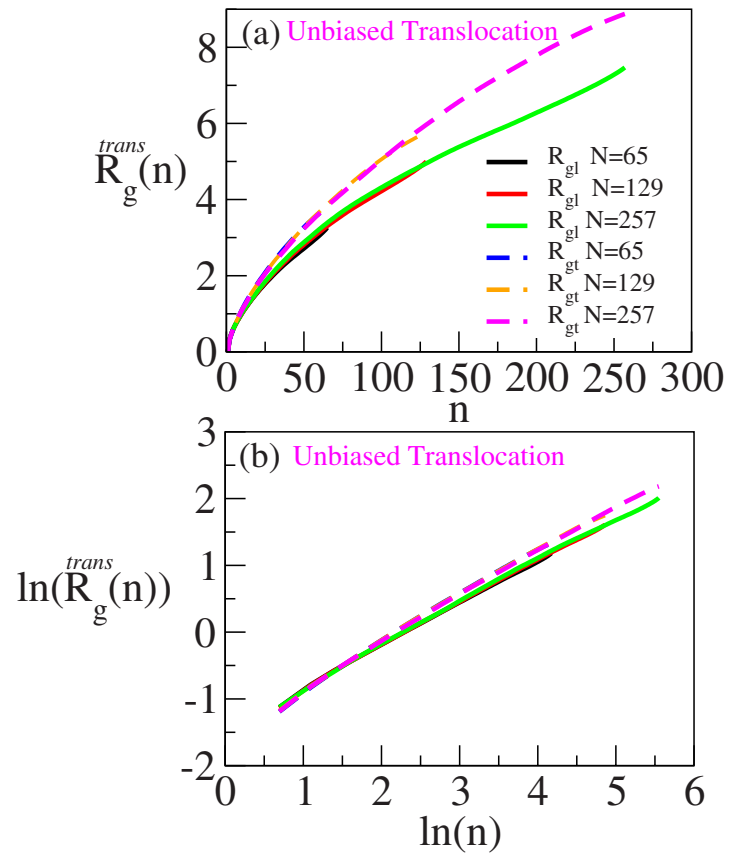

FIG. 14. (Color online) (a) Plot of longitudinal (solid lines) and transverse (dotted lines) components of gyration radii for the polymer which has just translocated as a function of the monomer index $m$ for unbiased translocation. The solid black, red, and green lines correspond to the longitudinal components and dotted blue, orange, and magenta lines correspond to the transverse components for chain lengths $N=65$ and 129 respectively. (b) The plot on a log-log scale.

of blobs of size $\xi(t)$. One expects that $\xi(t) \propto t^{1 / z}$ and then indeed there is a smooth crossover to the equilibrium size $R \propto N^{\nu}$ at $t=\tau$, with $\xi(\tau) \propto N^{\nu}$.

In a forced translocation process, the monomers move to the trans side so fast that the equilibrium structure cannot develop on the scale of the full chain, but only the first $N_{e q}$ monomers that have passed the pore can develop in their configuration the proper correlations due to excluded volume. We can estimate (for large enough $F$ ) this number $N_{e q}$ as follows. Following the same argument for the unbiased translocation we now need to replace $N$ by $N_{e q}$ so that the correlation length $\xi(\tau)$ at time $\tau$ is given by $\xi(\tau) \sim N_{e q}^{\nu}$ where that translocation time $\tau \sim N^{\alpha} / F$.

$$
\therefore N_{e q} \sim[\xi(\tau)]^{1 / \nu} \sim \tau^{\nu / z} \sim\left(\frac{N^{\alpha}}{F}\right)^{1 / \nu z} .
$$

Substituting $z=2+1 / \nu$ in above equation one gets

$$
N_{e q} \sim \xi(\tau)^{1 / \nu} \sim N^{\alpha /(2 \nu+1)} F^{-1 /(2 \nu+1)} .
$$

Therefore, the corresponding size $R$ is

$$
R \sim N_{e q}^{\nu} \sim \xi(\tau) \propto N^{\alpha \nu /(2 \nu+1)} F^{-1 /(2 \nu+1)}
$$

For $\alpha=1.36$ [26] we obtain $\alpha \nu /(2 \nu+1)=0.37$ while the value for $\alpha$ proposed in [4], $\alpha=1+\nu$ would yield $\alpha \nu /(2 \nu$ $+1)=0.43$. Of course, the estimate in Eq. (2), based on the $N_{e q}$ monomers that had enough time to equilibrate during the translocation time, is only a lower bound for the actual radius 
$R_{\text {trans }}$ of the translocated chain, because the additional $N$ $-N_{e q}$ monomers (translocated, but not yet equilibrated) can lead to some further increase in an uncorrelated way (so $R$ $\propto N^{1 / 2}$ probably is an upper bound). However, it remains a challenge to clarify whether the effective exponent $\nu_{\text {eff }}$ $\approx 0.45$ observed for several properties on the trans side (Figs. 6 and 10) really describes the asymptotic regime or is still affected by some correction to scaling effects; recall that in the data for unbiased translocation effective exponents vary from $\nu_{\text {eff }} \approx 0.55$ (Fig. 5) to $\nu_{\text {eff }} \approx 0.63$ (Fig. 1) around the true asymptotic value $\nu \approx 0.59$.

Another problem that is not understood is to explain the rather different behavior of $R_{g \ell}$ and $R_{g t}$ for large values of $m$ (close to $N$ ) in Fig. 13. The corresponding plot for the unbiasecase is shown in Fig. 14. These data are presumably dominated by the $N-N_{e q}$ monomers of the translocated chain that are still far from equilibrium. Therefore it is clear that the evolution of the translocated chain configuration with time (or with $m$, respectively) cannot be described in a simple manner.

Finally, it would be interesting to clarify the crossover between forced and unbiased translocation as $F \rightarrow 0$. Note that Eqs. (1) and (2) do not apply in this limit. Rather a simple scaling assumption could be

$$
\langle\tau\rangle=N^{2 \nu+1} f\left(F N^{2 \nu+1-\alpha}\right),
$$

where the scaling function $f(\zeta)$ behaves as $f(0)=$ const (unbiased translocation) while $f(\zeta \gg 1) \propto 1 / \zeta$ in order to recover Eq. (1) in the limit of large enough $F$. Figure 4 is a first indication that in the structure of the chain there is indeed an interesting and nontrivial dependence on $F$, but clearly more work is needed to fully clarify the aspects of this behavior by extensive simulations, which are very time consuming and therefore beyond the scope of the present work. But a safe general conclusion is that the theory of forced translocation needs to adequately account for the nonequilibrium aspects of the translocation process and the resulting strong asymmetry of the properties of the cis and trans parts of the chain.

\section{Distribution of the end to end length}

To get a better idea of the out of equilibrium aspects of the translocating chain we have looked at the distribution of the transverse and longitudinal components of the end to end vector $\mathbf{R}$ for the chain which has just translocated shown in Fig. 15. For comparison we have also shown the corresponding distribution of the equilibrated chain configurations located at the cis side at time $t=0$ at the beginning of each translocation process. One immediately notices that the distribution of $\mathbf{R}$ is much more restricted compared to its equilibrium configuration.

\section{SUMMARY AND DISCUSSION}

In summary, several aspects of forced polymer translocation are studied in this paper which explicitly point toward the out of equilibrium aspects of the translocation process. In several cases we also furnish data for the unbiased translocation for identical parameters of the simulation. From the
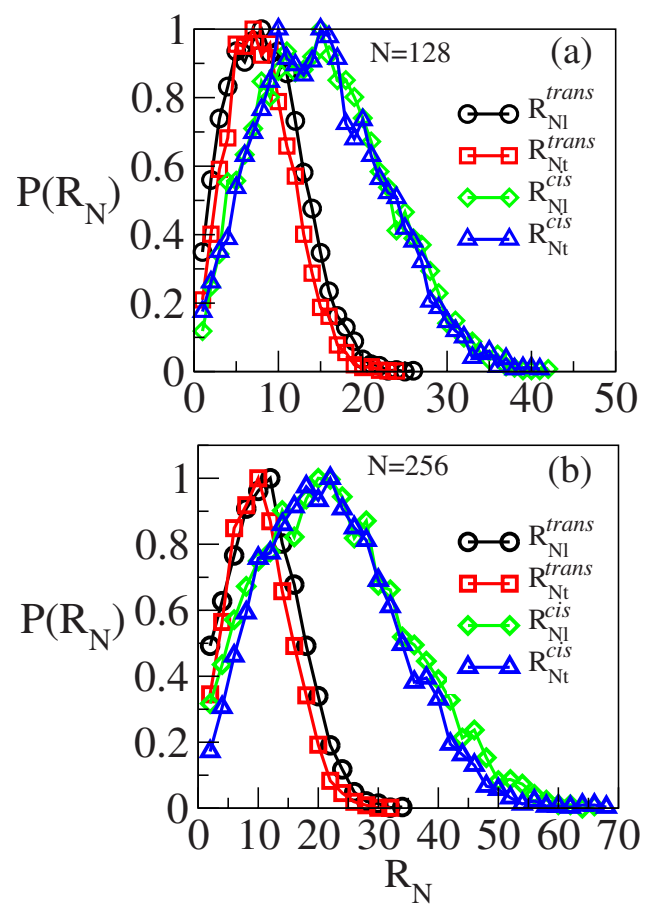

FIG. 15. (Color online) The distribution of longitudinal (circles) and transverse (squares) components of the end to end distances of a chain which has just translocated for chain lengths $N=128$ (top) and $N=256$ (bottom). For comparison the corresponding equilibrium configurations for the longitudinal (diamonds) and transverse (triangles) components at the cis side at the beginning of each translocation run are also shown.

series of data one could say that immediately after the translocation starts till it ends, the entire chain is always out of equilibrium. However, the nature and extent of this out of equilibrium aspect is different at the cis and trans sides and evolves with time. The "subchain" analysis vividly demonstrates this aspect in terms of subchain linear dimensions, with which we may associate the effective Flory exponent which is clearly largest in the immediate vicinity of the cis side of the pore, and acquires a smaller value in the in the immediate vicinity of the trans side of the pore. Immediately after the translocation process clearly the entire chain is compressed and described by a lower value of the effective Flory exponent. On the contrary the cis segments are described by a progressively higher value of the effective Flory exponent. In short, the bias at the pore makes the chain expanded and compressed at the cis and trans sides respectively, the chain tension being a function of the location of the segment (bead) with respect to the pore. This aspect was emphasized by Sakaue in a slightly different context. From the chain segment analysis we find that the translocation process can be viewed as a propagation of a "defect" in the value of the effective Flory exponent space in the opposite direction of translocation. Therefore, the analytic treatments in terms of one "slow" variable ( $s$ coordinate) is not adequate. Since the relaxation is different on the cis and trans side, one needs to incorporate additional slow variables to account for this trans-cis asymmetry [30]. We also present some elements of a phenomenological theoretical description of nonequilib- 


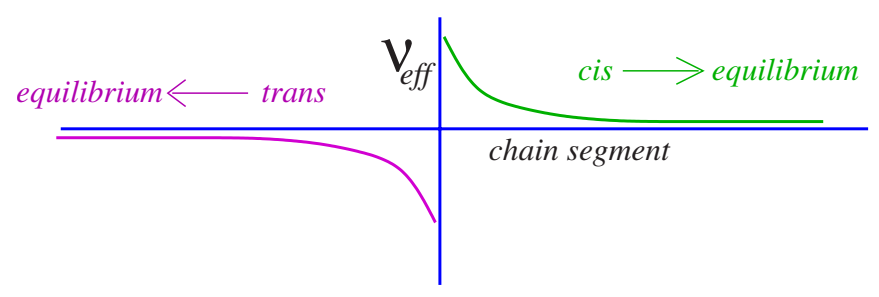

FIG. 16. (Color online) Contemplated defect schematic in the vicinity of the pore. The range of segment number $m$ over which a change in the effective exponent is observed increases with increasing size of the subchain that is considered.

rium behavior, in terms of an effective time-dependent correlation length that describes on which scales excluded volume interactions during translocation.

With the propagating defect picture in mind let us now look at the possible scenario in the limit of very large chain. In this limit Fig. 12 will look like as shown in Fig. 16, reaching the equilibrium value at each side farthest from the pore. How does the magnitude of the force dictate the correlation length of the decay of this defect? Furthermore, we know that in the limit of force $\rightarrow 0$ the segment analysis will remain valid and the defect will go to zero as there will be no distinction between the trans and cis side. Can one recover Kantor-Kardar result [4] from a finite-size chain simulation with proper extrapolation? While further work is needed to settle these issues we have initiated some work along this line in Sec. III H. We hope that the qualitative description of polymer translocation in terms of a defect in the value of the effective Flory exponent will motivate further theoretical work to construct a more complete theory of forced translocation.

\section{ACKNOWLEDGMENT}

A.B. appreciates the local hospitality at the Institut für Physik, Johannes Gutenberg Universität, Mainz, where most of this work was done and gratefully acknowledges the travel support from the Deutsche Forschungsgemeinschaft under Grant No. SFB 625/A3 and from the Schwerpunkt für Rechnergestützte Forschung in den Naturwissenschaften (SRFN). We also thank both the referees for their constructive comments.
[1] J. J. Kasianowicz, E. Brandin, D. Branton, and D. Deamer, Proc. Natl. Acad. Sci. U.S.A. 93, 13770 (1996); A. Meller, L. Nivon, E. Brandin, J. Golovchenko, and D. Branton, ibid. 97, 1079 (2000).

[2] J. L. Li, M. Gershow, D. Stein, E. Brandin, and J. A. Golovchenko, Nature Mater. 2, 611 (2003); A. J. Storm, J. H. Chen, X. S. Ling, H. W. Zandbergen, and C. Dekker, ibid. 2, 537 (2003).

[3] J. Chuang, Y. Kantor, and M. Kardar, Phys. Rev. E 65, 011802 (2001).

[4] Y. Kantor and M. Kardar, Phys. Rev. E 69, 021806 (2004).

[5] W. Sung and P. J. Park, Phys. Rev. Lett. 77, 783 (1996).

[6] M. Muthukumar, J. Chem. Phys. 111, 10371 (1999).

[7] J. L. A. Dubbeldam, A. Milchev, V. G. Rostiashvili, and T. A. Vilgis, Phys. Rev. E 76, 010801(R) (2007).

[8] J. L. A. Dubbeldam, A. Milchev, V. G. Rostiashvili, and T. A. Vilgis, Europhys. Lett. 79, 18002 (2007).

[9] J. K. Wolterink, G. T. Barkema, and D. Panja, Phys. Rev. Lett. 96, 208301 (2006).

[10] D. Panja, G. T. Barkema, and R. C. Ball, J. Phys.: Condens. Matter 19, 432202 (2007); 20, 075101 (2008).

[11] H. Vocks, D. Panja, G. T. Barkema, and R. C. Ball, J. Phys.: Condens. Matter 20, 095224 (2008).

[12] T. Sakaue, Phys. Rev. E 76, 021803 (2007).

[13] M. G. Gauthier and G. W. Slater, J. Chem. Phys. 128, 065103 (2008); 128, 205103 (2008).

[14] A. Milchev, K. Binder, and A. Bhattacharya, J. Chem. Phys. 121, 6042 (2004).

[15] K. Luo, T. Ala-Nissila, and S.-C. Ying, J. Chem. Phys. 124, 034714 (2006).

[16] K. Luo, T. Ala-Nissila, and S.-C. Ying, J. Chem. Phys. 124, 114704 (2006).

[17] I. Huopaniemi, K. Luo, T. Ala-Nissila, and S.-C. Ying, J.
Chem. Phys. 125, 124901 (2006).

[18] D. Wei, W. Yang, X. Jin, and Q. Liao, J. Chem. Phys. 126, 204901 (2007).

[19] K. Luo, T. Ala-Nissila, S.-C. Ying, and A. Bhattacharya, J. Chem. Phys. 126, 145101 (2007); Phys. Rev. Lett. 99, 148102 (2007); 100, 058101 (2008).

[20] S. Matysiak, A. Montesi, M. Pasquali, A. B. Kolomeisky, and C. Clementi, Phys. Rev. Lett. 96, 118103 (2006).

[21] S. Guillouzic and G. W. Slater, Phys. Lett. A 359, 261 (2006).

[22] M. G. Gauthier and G. W. Slater, Eur. Phys. J. E 25, 17 (2008).

[23] S. Fugmann and I. M. Sokolov, Phys. Rev. E 79, 021803 (2009).

[24] K. Luo, S. T. T. Ollila, I. Huopaniemi, T. Ala-Nissila, P. Pomorski, M. Karttunen, S.-C. Ying, and A. Bhattacharya, Phys. Rev. E 78, 050901(R) (2008).

[25] V. V. Lehtola, R. P. Linna, and K. Kaski, Europhys. Lett. 85, 58006 (2009); Phys. Rev. E 78, 061803 (2008); See also K. Luo, T. Ala-Nissila, S.-C. Ying, and R. Metzler, Eur. Phys. Lett. 88, 68006 (2009).

[26] A. Bhattacharya, W. H. Morrison, K. Luo, T. Ala-Nissila, S.-C. Ying, A. Milchev, and K. Binder, Eur. Phys. J. E 29, 423 (2009).

[27] J. L. A. Dubbeldam, A. Milchev, V. G. Rostiashvili, and T. A. Vilgis, J. Phys.: Condens. Matter 21, 098001 (2009).

[28] G. S. Grest and K. Kremer, Phys. Rev. A 33, 3628 (1986).

[29] For the case of unbiased translocation the cis/trans components are defined if the chain translocates from left ( $\mathrm{cis}$ ) to right (trans) or vice versa unlike the case of forced translocation where the applied bias uniquely defines the cis and the trans components.

[30] A.B. gratefully acknowledges the conversation with W. Sung on this particular aspect of the translocation problem. 